\title{
84. NUEVAS APORTACIONES A LA FLORA DE ANDALUCÍA: SIERRA DE GÁDOR, ALMERÍA (ANDALUCÍA, ESPAÑA). NOTA II.
}

\author{
Esther GIMÉNEZ, Juan Francisco MOTA, Francisco GÓMEZ MERCADO, \\ Pedro SORIA, Miguel CUETO y Antonio M. AGUILERA
}

\begin{abstract}
New floristic records in Andalusia: Sierra de Gádor, Almeria (Andalucía, Spain). Note II.
Palabras clave. Flora, corología, Almería, Andalucía.
\end{abstract}

Key words. Flora, corology, Almería, Andalusia.

Como resultado de las numerosas campañas de herborización de la flora de la Sierra de Gádor (Almería), comunicamos la presencia de doce novedades florísticas para el territorio, algunas de ellas lo son para la provincia de Almería. Aportamos además datos sobre su ecología y corología. La nomenclatura de los taxones tratados está basada en Flora Ibérica (Castroviejo et al. eds. 1986-2001) y Flora Europaea (Tutin et al. eds. 1964-1980) excepto el género Centaurea (García Jacas, 1998). Los pliegos testigo han sido depositados en el Herbario de la Universidad de Almería (ALMEU), para taxon se indica la localidad, ecología, aproximación fitosociológica basada en Rivas Martínez et al. (1999) y el número de pliego registrado.

Astragalus alopecuroides L. subsp. alopecuroides ALMERÍA: Sierra de Gádor, Los Ortices, 30SWF0788, $1300 \mathrm{~m}$, matorral heliófilo, Rosmarinetalia officinalis, 8-VI-97, E. Giménez \& F. Gómez Mercado, AlmeU 1393. Ampliamos la corología de este taxon ibérico que no había sido citado por Podlech (2000:303) para la provincia de Almería. Hasta el momento en la Sierra de Gádor conocemos únicamente esta población que presenta un número de individuos reducido.

\section{Centaurea gabrielis-blancae Fdez. Casas}

ALMERÍA: Sierra de Gádor, Loma de la Chanata, 30SWF2687, $1350 \mathrm{~m}$, matorral heliófilo, Lavandulo-Genistion boissieri, 25/VI/02, E. Giménez \& M. Cueto, ALMEU 2338. Endemismo de la Península Ibérica con amplia distribución altitudinal. Primera cita para la Sierra de Gádor.

\section{Centaurea kunkelii García-Jacas}

ALMERÍA: Sierra de Gádor, Loma de las Ventillas, 30SWF0885, $1350 \mathrm{~m}$, matorral de cunetas, Lavandulo-Genistion boissieri, 9/VII/98, E. Giménez \& F. Gómez-Mercado, ALMEU 2191. Endemismo de la Sierra de Gádor, hasta ahora las poblaciones conocidas corresponden a dos localidades próximas del termino municipal de Enix, citadas en el trabajo en el que se describió el taxon (García Jacas, 1998). Hemos localizado una nueva población en la Loma de las Ventillas, con un número de individuos bastante reducido.

\section{Centranthus nevadensis Boiss.}

ALMERÍA: Sierra de Gádor, Tajos de la Parra, 30SWF1289, $1680 \mathrm{~m}$, comunidades de paredones verticales, Saxifragion camposii, 19/VII/01, E. Giménez \& A. Aguilera, ALMEU 2352. Endemismo esencialmente nevadense, aunque de modo testimonial se ha localizado en la Sierra de Tejeda (Granada), Sierra de Grazalema (Cádiz) (Blanca, 2001), Sierra de los Filabres (Peñaset al., 2001), Sierra de las Nieves (Málaga) (Pérez Latorre et al., 1998). Con esta nueva cita ampliamos su distribución a la Sierra de Gádor (Almería).

Conringia orientalis (L.) Dumort.

ALMERÍA: Sierra de Gádor, Caparidán, 30SWF0887, $1600 \mathrm{~m}$, herbazales nitrófilos, Centaureetalia cyani, 20-V-02, E. Giménez \& M. Cueto, ALMEU 2344. Elemento de amplia distribución, en la Península Ibérica se distribuye por el sur y el este. Fernandes (1993) la cita como especie dudosa 
en la provincia de Almería. Con esta nueva referencia confirmamos su presencia en la provincia y la citamos como novedad para la Sierra de Gádor.

\section{Cynoglossum nebrodense Guss.}

ALMERÍA: Sierra de Gádor, Barranco del Chaparral, 30SWF1685, $1700 \mathrm{~m}$, nitrófila de montaña, Onopordetalia acanthii, 7/VII/02, P. Soria \& J.F. Mota, ALMEU 2351. Endemismo de las Sierras Béticas. Anteriormente había sido citado para la provincia de Almería en la Sierra de María (Cueto \& Blanca, 1997).

Delphinium halteratum $\mathrm{Sm}$. subsp. verdunense (Balbis) Graebner \& Graebner fil.

ALMERÍA: Sierra de Gádor, Bco. de los Infantes, 30SWF0871, $335 \mathrm{~m}$, bordes de caminos, Roemerion hybridae, 6/VI/02, E. Giménez, J. Cabello, D. Alcaraz \& A. Aguilera, ALMEU 2346. Este taxon había sido citado en la Sierra de Gádor (Pallarés in Sagredo, 1987), aunque su presencia en la provincia de Almería no es reconocida por Blanché \& Molero (1986:250). Confirmamos su presencia con esta nueva cita.

\section{Echium flavum Desf.}

ALMERÍA: Sierra de Gádor, Puntal de los Pájaros, 30SWF1685, $2100 \mathrm{~m}$, matorral orófilo algo nitrificado, 4/VII/88, P. Soria, ALMEU 2349. Elemento iberonorteafricano que hasta el momento no se había citado para la Sierra de Gádor. En la provincia de Almería aparece en las zonas altimontanas de la Sierra de María (Cueto \& Blanca, 1997).

\section{Erinus alpinus L.}

ALMERÍA: Sierra de Gádor, Bco. del Chaparral, 30SWF1785, $1700 \mathrm{~m}$, comunidades de paredones verticales umbríos, Saxifragion camposii, 7/VII/02, P. Soria \& J.F. Mota, ALMEU 2350. Este taxon de distribución amplia se presenta en los roquedos y paredones verticales umbríos de las sierras béticas, en la provincia de Almería solo se había citado de la Sierra de María (Cueto \& Blanca, 1997).

\section{Lathyrus pulcher J. Gay}

ALMERÍA: Sierra de Gádor, Los Ortices, 30SWF0788, $1300 \mathrm{~m}$, comunidades herbáceas de cunetas húmedas, Brachypodion phoenicoidis, 20V-02, E. Giménez \& M. Cueto, AlMEU 2342.
Elemento iberonorteafricano que en la Península Ibérica se distribuye por el E y SE, sin embargo no se había reconocido su presencia en la provincia de Almería (Gallego, 1999:442).

\section{Scleranthus polycarpus L.}

ALMERÍA: Sierra de Gádor, Balsa del Sabinar, 30SWFI282, $1850 \mathrm{~m}$, pastizal terofítico, Tuberarietea guttatae, 20-V-02, E. Giménez \& M. Cueto, ALMEU 2341. Pequeño terófito que se distribuye por Europa, $\mathrm{N}$ de África y Asia Menor. Aunque en la Península Ibérica aparece de forma dispersa por la mayoría del territorio, no se había citado su presencia en la Sierra de Gádor.

\section{Vincetoxicum nigrum (L.) Moench}

ALMERÍA: Sierra de Gádor, Bco. de la Atalaya, 30SWF2081, $1700 \mathrm{~m}$, herbazal húmedo bajo aceral, Quercetalia ilicis, 20/V/91. P. Soria, ALMEU 2348. Esta hierba perenne de distribución amplia se presenta en zonas con humedad ambiental y casi siempre se presenta resguardada bajo bosques o matorrales de mayor porte, en la Sierra de Gádor se presenta bajo la protección del aceral. En la provincia de Almería tan solo se había citado de la Sierra de María (Cueto \& Blanca, 1997).

\section{BIBLIOGRAFÍA}

BLANCA, G. -2001- Flora en García Canseco, V (coord.) Parque Nacional de Sierra Nevada. Canseco Editores.

BLANCHÉ, C. y J. MOLERO -1986- Delphinium L. en Castroviejo, S. et al. (eds.) Flora Ibérica I: Lycopodiaceae-Papaveraceae. Real Jardín Botánico, CSIC. Madrid.

CASTROVIEJO, S. et al., eds. -1986-2001- Flora Ibérica, Vols. I-VIII. CSIC. Real Jardín Botánico de Madrid. Madrid.

CUETO, M. y G. BLANCA -1997-Flora del Parque Natural Sierra de María-Los Vélez. Ed. SAHN. Almería.

FERNANDES, R.B. -1993- Conringia Heist. ex Fabr. en Castroviejo, S. et al. (eds.) Flora Ibérica IV: Cruciferae-Monotropaceae. Real Jardín Botánico, CSIC. Madrid.

GALLEGO, M.J. -1999- Lathyrus L. en Talavera, S. et al. (eds.) Flora Ibérica VII(I): Leguminosae. Real Jardín Botánico, CSIC. Madrid. 
GARCÍA JACAS, N. -1998- Centaurea kunkelii (Asteraceae, Carducae), a new hybridogenic endecaploid species of sect. Acrocentro from Spain. Ann. Bot. Fennici 35:159-167.

PEÑAS, J., J. CABELLO, F. VALLE TENDERO \& J.F. MOTA -2001-Comunidades vegetales rupícolas y subrupícolas del sudeste ibérico (Sierra de los Filabres). Lazaroa 22:95-107.

PÉREZ LATORRE, A.V., P. NAVAS, D. NAVAS, Y. GIL y B. CABEZUDO -1998- Datos sobre la flora y vegetación de la Serranía de Ronda (Málaga, España). Acta Bot. Malacitana 23:149191

PODLECH, D. -2000- Astragalus L. en Talavera, S. et al. (eds.) Flora Ibérica VII(II): Leguminosae. Real Jardín Botánico, CSIC. Madrid.
RIVAS MARTÍNEZ, S., F. FERNÁNDEZ GONZÁLEZ \& J. LOIDI ARREGUI -1999- Checklist of plant communities of Iberian Peninsula, Balearic and Canary Islands to suballiance level. Itinera Geobotania 13:353-451.

SAGREDO, R. -1987- Flora de Almería. IEA.

TUTIN, T.G. et al., eds. -1964-1980- Flora Europaea. Vols. 1-5. Cambridge Univ. Press, Cambridge.

Aceptado para su publicación en septiembre de 2002

Dirección de los autores. Dpto. Biología Vegetal y Ecol. Escuela Politécnica Superior. Univ. de Almería. 04120 Almería.

\section{NUEVAS APORTACIONES AL CONOCIMIENTO DE LA FLORA DE LA SIERRA DE LÍBAR ( PARQUE NATURAL SIERRA DE GRAZALEMA, MÁLAGA)}

Manuel BECERRA PARRA

New records for the flora of Sierra de Líbar (Grazalema Natural Park, Málaga province)

Palabras clave. Flora, Parque Natural Sierra de Grazalema, Málaga, Sierra de Líbar.

Key words. Flora, Natural Park, Grazalema, Málaga, Sierra de Líbar.

Con objeto de aportar datos para el mejor conocimiento de la flora vascular de la provincia de Málaga, hemos realizado herborizaciones en las Sierras de Líbar y Grazalema. La Sierra de Líbar se ubica en la zona suroccidental de la Serranía de Ronda y dentro de la cuenca alta del río Guadiaro. Ocupa los municipios malagueños de Cortes de la Frontera, Jimera de Líbar, Benaoján y
Montejaque, y el gaditano de Villaluenga del Rosario. Las altitudes oscilan entre $\operatorname{los} 300$ y los 1.400 m.s.m., estando el clima muy influenciado por las elevadas precipitaciones anuales que se registran, las cuales llegan a alcanzar los $2.000 \mathrm{~mm}$ anuales en las cumbres más elevadas. Los materiales de ambas sierras son en su mayoría rocas sedimentarias de naturaleza carbonatada, principalmente calizas, 